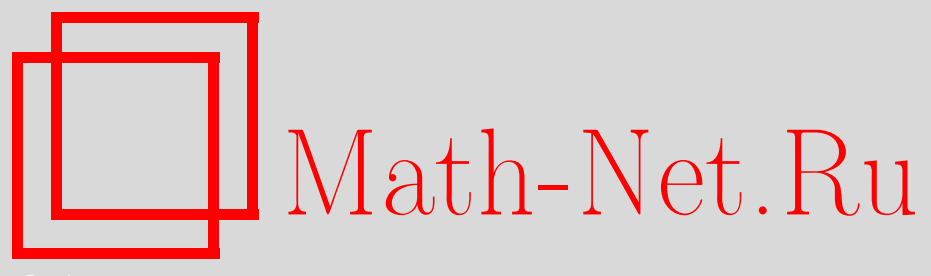

Л. Г. Арабаджян, Об одной бесконечной алгебраической системе в нерегулярном случае, Матем. заметки, 2011, том 89, выпуск 1, 3-11

DOI: https://doi.org/10.4213/mzm6578

Использование Общероссийского математического портала Math-Net.Ru подразумевает, что вы прочитали и согласны с пользовательским соглашением http://www .mathnet.ru/rus/agreement

Параметры загрузки:

IP : 54.237 .206 .68

26 апреля 2023 г., 12:49:25

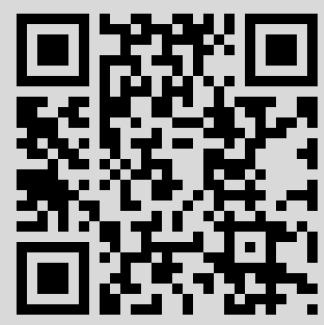


Том 89 выпуск 1 январь 2011

УДК 517

\section{Об одной бесконечной алгебраической системе в нерегулярном случае}

\section{Л. Г. Арабаджян}

В работе получены достаточные условия нетривиальной разрешимости систем вида

$$
\varphi_{i}=b_{i}+\lambda_{i} \sum_{j=0}^{\infty} a_{i-j} \varphi_{j}, \quad i \in \mathbb{Z}_{+} \stackrel{\text { def }}{=}\{0,1,2, \ldots, n, \ldots\},
$$

и соответствующих однородных систем. Предполагается, что данные последовательности $b=\left(b_{0}, b_{1}, b_{2}, \ldots\right), \lambda=\left(\lambda_{0}, \lambda_{1}, \lambda_{2}, \ldots\right)$ и данная теплицевая матрица $A=\left(a_{i-j}\right)$ удовлетворяют условиям

$$
\begin{array}{ll}
a_{j} \geqslant 0, \quad j \in \mathbb{Z}, \quad & \sum_{j=-\infty}^{\infty} a_{j}=1, \quad \sum_{j=-\infty}^{\infty}|j| a_{j}<\infty, \quad \sum_{j=-\infty}^{\infty} j a_{j}<0, \\
b_{j} \geqslant 0, \quad j \in \mathbb{Z}_{+}, & \sum_{j=0}^{\infty} b_{j}<\infty, \quad 1 \leqslant \lambda_{i} \leqslant\left(\sum_{j=-\infty}^{i} a_{j}\right)^{-1}, \quad i \in \mathbb{Z}_{+} .
\end{array}
$$

При выполнении указанных условий построены ограниченные решения однородной и неоднородной систем указанного выше вида.

Библиография: 8 названий.

Рассмотрим систему вида

$$
f_{i}=b_{i}+\sum_{j=0}^{\infty} c_{i j} f_{j}, \quad i \in \mathbb{Z}_{+} \stackrel{\text { def }}{=}\{0,1,2, \ldots, n, \ldots\},
$$

относительно искомой последовательности $f=\left(f_{0}, f_{1}, f_{2}, \ldots\right)$. Системы такого вида возникают в приложениях и представляют известный теоретический и прикладной интерес. Наиболее интересные и сложные ситуации возникают в особых случаях, когда необратима матрица $I-C$, где $C=\left(c_{i j}\right)_{i, j=0}^{\infty}$, а $I-$ бесконечная единичная матрица. Результаты общего характера, связанные с разрешимостью системы (1), содержатся в [1], [2].

В [2] введены понятия регулярных и вполне регулярных систем вида (1): регулярные системы характеризуются условиями

$$
q_{i} \stackrel{\text { def }}{=} \sum_{j=0}^{\infty}\left|c_{i j}\right|<1, \quad i \in \mathbb{Z}_{+}
$$


вполне регулярные системы - условиями

$$
q_{i} \leqslant q<1, \quad i \in \mathbb{Z}_{+}
$$

Для регулярных систем (1) в работе [2] получены достаточные условия существования ограниченного решения $f=\left(f_{0}, f_{1}, f_{2}, \ldots\right) \in m$. А именно, система (1) в случае $(\delta 1)$ обладает ограниченным решением, если величины $b_{i}$ удовлетворяют условиям

$$
\left|b_{1}\right| \leqslant k\left(1-q_{i}\right), \quad i \in \mathbb{Z}_{+},
$$

при котором $k>0$.

Возникает вопрос изучения системы (1) в тех случаях, когда условия регулярности $(\delta 1)$ нарушаются. Заметим, что в случае теплицевых матриц $C=\left(c_{i-j}\right)$ удается значительно ослабить требования на матрицу и свободный член (см. [3], [4]).

Случай, когда имеют место соотношения

$$
c_{i j} \geqslant 0, \quad q_{i}=1, \quad i, j \in \mathbb{Z}_{+},
$$

назовем ко-консервативным (co-conservative). Возможность получения результатов в данном направлении на основе треугольной факторизации матрицы $C$ дается теоремой 7.4 работы [5]. Для двусторонней бесконечной ко-консервативной системы

$$
\widehat{f_{i}}=b_{i}+\sum_{j=-\infty}^{\infty} a_{i-j} \widehat{f}_{j}, \quad i \in \mathbb{Z} \stackrel{\text { def }}{=}\{0, \pm 1, \pm 2, \ldots,\},
$$

вопросы разрешимости изучены в [6] посредством факторизации теплицевой матрицы $A=\left(a_{i-j}\right)_{i, j=-\infty}^{\infty}$ на основе результатов работы [7].

В настоящей работе рассматривается определенный класс ко-консервативных систем вида (1), обладающих положительным решением. Получены достаточные условия существования нетривиальных неотрицательных решений систем вида

$$
\varphi_{i}=b_{i}+\lambda_{i} \cdot \sum_{j=0}^{\infty} a_{i-j} \varphi_{j}, \quad i \in \mathbb{Z}_{+},
$$

относительно искомой последовательности $\varphi=\left(\varphi_{0}, \varphi_{1}, \varphi_{2}, \ldots\right)$ и соответствующих однородных систем. Предполагается, что последовательности $b=\left(b_{0}, b_{1}, b_{2}, \ldots\right)$ и $\lambda=\left(\lambda_{0}, \lambda_{1}, \lambda_{2}, \ldots\right)$, а также теплицевая матрица $A=\left(a_{i-j} t\right)_{i, j=0}^{\infty}$ удовлетворяют условиям

$$
\begin{gathered}
a_{j} \geqslant 0, \quad j \in \mathbb{Z}, \\
\sum_{j=-\infty}^{\infty} a_{j}=1, \\
\sum_{j=-\infty}^{\infty}|j| a_{j}<\infty, \\
\nu=\sum_{j=-\infty}^{\infty} j a_{j}<0,
\end{gathered}
$$




$$
\begin{gathered}
1 \leqslant \lambda_{k} \leqslant\left(\sum_{j=-\infty}^{k} a_{j}\right)^{-1}, \quad k=0,1,2, \ldots, \\
b_{k} \geqslant 0, \quad k \in \mathbb{Z}_{+}, \\
\sum_{j=0}^{\infty} b_{j}<+\infty .
\end{gathered}
$$

Системы вида (2) возникают в некоторых задачах теории марковских цепей.

1. Разрешимость однородной системы. Наряду с (2) рассмотрим однородную систему

$$
\psi_{i}=\lambda_{i} \cdot \sum_{j=0}^{\infty} a_{i-j} \psi_{j}, \quad i \in \mathbb{Z}_{+},
$$

где матрица $A=\left(a_{i-j}\right)$ и последовательность $\lambda=\left(\lambda_{0}, \lambda_{1}, \lambda_{2}, \ldots\right)$ удовлетворяют условиям $(\alpha 1)-(\alpha 4)$ и $(\beta 1)$, и изучим вопросы ее нетривиальной разрешимости. В частном случае при $\lambda_{i}=1, i \in \mathbb{Z}_{+}$из системы (3) получается дискретное (однородное) уравнение Винера-Хопфа

$$
\eta_{i}=\sum_{j=0}^{\infty} a_{i-j} \eta_{j}, \quad i \in \mathbb{Z}_{+} .
$$

Заметим, что при выполнении условий $(\alpha 1),(\alpha 2)$ и $a_{k} \neq 0$ для всех $k \in \mathbb{Z}$ система (4) является регулярной, но не вполне регулярной, ибо в этом случае имеем

$$
q_{i}=\sum_{j=0}^{\infty}\left|c_{i j}\right|=\sum_{j=0}^{\infty} a_{i-j}=\sum_{k=-\infty}^{i} a_{k}<1, \quad i \in \mathbb{Z}_{+} .
$$

При тех же условиях и при $\lambda_{k}=\left(\sum_{j=-\infty}^{k} a_{j}\right)^{-1}$ для всех $k \in \mathbb{Z}_{+}$системы (2) и (3) являются ко-консервативными в силу соотношений

$$
q_{i}=\lambda_{i} \cdot \sum_{j=0}^{\infty} a_{i-j}=\lambda_{i} \cdot \sum_{p=-\infty}^{i} a_{p}=1, \quad i \in \mathbb{Z}_{+} .
$$

Вопросы разрешимости системы (4) и соответствующей неоднородной системы

$$
\zeta_{i}=b_{i}+\sum_{j=0}^{\infty} a_{i-j} \zeta_{j}, \quad i \in \mathbb{Z}_{+},
$$

при выполнении $(\alpha 1),(\alpha 2)$ на основе треугольной факторизации матрицы $I-A$ изучались в работе [3]. В этой работе построено, в частности, положительное, ограниченное и возрастающее решение $\eta=\left(\eta_{0}, \eta_{1}, \eta_{2}, \ldots\right)$ системы (4) в случае $(\alpha 1),(\alpha 2)$ при дополнительных условиях $(\alpha 3),(\alpha 4)$. Соответствующее решение, удовлетворяющее условию $\eta_{0}=1$, обозначим через $\eta^{*}: \eta^{*}=\left(1, \eta_{1}^{*}, \eta_{2}^{*}, \ldots\right)$.

$1^{\circ}$. Введем обозначение

$$
\widehat{\eta}=\sup _{1 \leqslant k<\infty} \eta_{k}^{*}
$$

Очевидно, что $1 \leqslant \widehat{\eta}<\infty$. 
Займемся вопросами разрешимости системы (3) при $(\alpha 1)-(\alpha 4)$ и ( $\beta 1)$. Рассмотрим итерации

$$
\psi_{i}^{(n+1)}=\lambda_{i} \cdot \sum_{j=0}^{\infty} a_{i-j} \psi_{j}^{(n)}, \quad \psi_{i}^{(0)}=\widehat{\eta}, \quad i \in \mathbb{Z}_{+}, \quad n=1,2,3 \ldots,
$$

где величина $\widehat{\eta}$ определяется согласно (6). Покажем, что эти итерации монотонно убывают по $n$ и снизу ограничены величинами $\eta_{i}^{*}, i \in \mathbb{Z}_{+}$. Действительно, при $n=0$ из (7) для всех $i \in \mathbb{Z}_{+}$имеем

$$
\psi_{i}^{(1)}=\lambda_{i} \cdot \sum_{j=0}^{\infty} a_{i-j} \widehat{\eta}=\widehat{\eta} \cdot \lambda_{i} \cdot \sum_{k=-\infty}^{i} a_{k} \leqslant \widehat{\eta}=\psi_{i}^{(0)}, \quad \text { т.e. } \quad \psi_{i}^{(1)} \leqslant \psi_{i}^{(0)} .
$$

Допустим, что для некоторого $n \in N$ и для всех $i \in \mathbb{Z}_{+}$имеют место неравенства $\psi_{i}^{(n)} \leqslant \psi_{i}^{(n-1)}$. Тогда из $(7)$ получаем

$$
\psi_{i}^{(n+1)}=\lambda_{i} \cdot \sum_{j=0}^{\infty} a_{i-j} \psi_{j}^{(n)} \leqslant \lambda_{i} \cdot \sum_{j=0}^{\infty} a_{i-j} \psi_{j}^{(n-1)}=\psi_{i}^{(n)} .
$$

Таким образом, для всех $i \in \mathbb{Z}_{+}$и $n=0,1,2, \ldots$ имеем $\psi_{i}^{(n)} \leqslant \psi_{i}^{(n-1)}$. Рассмотрим величины $\psi_{i}^{(n)}$ при фиксированном $i \in \mathbb{Z}_{+}$. Очевидно, что $\psi_{i}^{(n)} \downarrow$ по $n$. С другой стороны, используя соотношения (7), методом математической индукции можно доказать справедливость оценок

$$
\psi_{i}^{(n)} \geqslant \eta_{i}^{*}, \quad i \in \mathbb{Z}_{+}, \quad n=0,1,2, \ldots
$$

Итак, при любом фиксированном $i \in \mathbb{Z}_{+}$монотонно убывающая последовательность $\left\{\psi_{i}^{(n)}\right\}$ ограничена снизу. Поэтому существует предел $\lim _{n \rightarrow \infty} \psi_{i}^{(n)}=\psi_{i}$. Рассмотрим предельную последовательность $\psi=\left(\psi_{0}, \psi_{1}, \psi_{2}, \ldots\right)$. Поскольку ряд правой части равенства (7) сходится равномерно по $n$, можно в этом равенстве перейти к пределу при $n \rightarrow \infty$. В результате получаем равенства (3) относительно предельных величин $\psi_{i}, i \in \mathbb{Z}_{+}$.

Таким образом, доказана

Теорема 1. Пусть в системе (3) матрица $A=\left(a_{i-j}\right)$ удовлетворяет условиям $(\alpha 1)-(\alpha 4)$, а последовательность $\lambda=\left(\lambda_{0}, \lambda_{1}, \lambda_{2}, \ldots\right)$ - условию $(\beta 1)$. Тогда эта система обладает ограниченным нетривиальным решением $\psi=\left(\psi_{0}, \psi_{1}, \psi_{2}, \ldots\right)$ таким, что $\psi_{i} \geqslant \eta_{i}^{*}$ для всех $i \in \mathbb{Z}_{+}$, где $\eta^{*}=\left(1, \eta_{1}^{*}, \eta_{2}^{*}, \ldots\right)$ - рассмотренное выше решение системы (4).

$2^{\circ}$. На основе результатов работ [3], [4], [6] при некоторых дополнительных ограничениях на величины $\lambda_{i}$ можно доказать существование нетривиального неотрицательного решения системы (3) в случае, когда выполняются условия $(\alpha 1)-(\alpha 4)$ и $0 \leqslant \lambda_{i} \leqslant 1, i \in \mathbb{Z}_{+}$. В работе [8] рассматривалась подобная задача для непрерывного аналога системы (3).

Рассмотрим систему

$$
\varphi_{i}^{*}=\left(1-\lambda_{i}^{*}\right) \eta_{i}^{*}+\lambda_{i}^{*} \cdot \sum_{j=0}^{\infty} a_{i-j} \varphi_{i}^{*}, \quad i \in \mathbb{Z}_{+},
$$


где матрица $A=\left(a_{i-j}\right)$ удовлетворяет условиям $(\alpha 1)-(\alpha 4), \eta^{*}=\left(1, \eta_{1}^{*}, \eta_{2}^{*}, \ldots\right)-$ вышеуказанное (положительное и ограниченное) решение системы (4) с той же матрицей $A$, а последовательность $\lambda^{*}=\left(\lambda_{0}^{*}, \lambda_{1}^{*}, \lambda_{2}^{*}, \ldots\right)$ удовлетворяет условиям

$$
0 \leqslant \lambda_{i}^{*} \leqslant 1, \quad i \in \mathbb{Z}_{+}, \quad \sum_{j=0}^{\infty}\left(1-\lambda_{i}^{*}\right)<+\infty .
$$

Очевидно, что в этом случае имеем

$$
\sum_{i=0}^{\infty}\left(1-\lambda_{i}^{*}\right) \eta_{i}^{*}<+\infty
$$

Система (8) имеет очевидное решение $\varphi_{i}^{*}=\eta_{i}^{*}, i \in \mathbb{Z}_{+}$. Ниже будет доказано, что эта же система обладает также другим решением $\widetilde{\varphi}^{*}=\left(\widetilde{\varphi}_{0}^{*}, \widetilde{\varphi}_{1}^{*}, \widetilde{\varphi}_{2}^{*}, \ldots\right)$ со свойствами

$$
\widetilde{\varphi}_{i}^{*} \geqslant 0, \quad i \in \mathbb{Z}_{+} \quad \widetilde{\varphi}_{i}^{*} \rightarrow 0, \quad i \rightarrow \infty .
$$

С этой целью рассмотрим неоднородную систему (5) и систему

$$
\rho_{i}=b_{i}+\lambda \cdot \sum_{j=0}^{\infty} a_{i-j} \rho_{j}, \quad i \in \mathbb{Z}_{+},
$$

с той же, что и в $(8)$, матрицей $A=\left(a_{i-j}\right)$ и величинами $\lambda_{i}$, удовлетворяющими условиям $0 \leqslant \lambda_{i} \leqslant 1, i \in \mathbb{Z}_{+}$. Система (5) будет мажорантной для системы (10) (см. [2]). Согласно теореме 1 работы [2] в случае существования неотрицательного решения $\zeta=\left(\zeta_{0}, \zeta_{1}, \zeta_{2}, \ldots\right), \zeta_{i} \geqslant 0, i \in \mathbb{Z}_{+}$, системы (5), система (10) также обладает решением $\rho=\left(\rho_{0}, \rho_{1}, \rho_{2}, \ldots\right)$, которое может быть найдено методом последовательных приближений и для которого в случае $b_{i} \geqslant 0, i \in \mathbb{Z}_{+}$справедливы неравенства

$$
0 \leqslant \rho_{i} \leqslant f_{i}, \quad i \in \mathbb{Z}_{+} .
$$

Теперь рассмотрим систему (5) и докажем, что при условиях $(\alpha 1)-(\alpha 4), b_{i} \geqslant 0$ и $\sum_{j=0}^{\infty} b_{i}<+\infty$ эта система обладает решением $\zeta=\left(\zeta_{0}, \zeta_{1}, \zeta_{2}, \ldots\right)$ со свойствами

$$
\zeta_{i} \geqslant 0, \quad i \in \mathbb{Z}_{+}, \quad \zeta_{i} \rightarrow 0, \quad i \rightarrow \infty .
$$

В работе [7] (см. также [3], [4]), для решения систем вида (5) был разработан оригинальный метод, основанный на треугольной факторизации матрицы $I-A$, где $I$ - бесконечная единичная матрица.

Пусть $A=\left(a_{i-j}\right)$ - теплицевая матрица, удовлетворяющая условиям $(\alpha 1),(\alpha 2)$. Тогда существуют (см. [7]) такие треугольные теплицевые матрицы $X=\left(x_{i-j}\right)$, $x_{k}=0$ при $k<0$ и $Y=\left(y_{i-j}\right), y_{k}=0$ при $k<0$, что

$$
I-A=(I-Y)(I-X) .
$$

В [3] доказано, в частности, что если к условиям $(\alpha 1),(\alpha 2)$ добавить условия $(\alpha 3)$, $(\alpha 4)$, то матрицы $X$ и $Y$ будут обладать свойствами

$$
x_{j} \geqslant 0, \quad y_{j} \geqslant 0, \quad \sigma_{-} \stackrel{\text { def }}{=} \sum_{j=0}^{\infty} y_{j}=1, \quad \sigma_{+} \stackrel{\text { def }}{=} \sum_{j=0}^{\infty} x_{j}<1,
$$

что означает необратимость $I-Y$ и обратимость $I-X$ любом из пространств $l_{1}, m, c$. 
Факторизация (13) сводит решение системы [5] к последовательному решению следующих двух систем:

$$
\begin{aligned}
h_{i}=b_{i}+\sum_{j=i}^{\infty} y_{j-i} h_{j}, & i \in \mathbb{Z}_{+}, \\
f_{i}=h_{i}+\sum_{j=0}^{i} x_{i-j} f_{j}, & i \in \mathbb{Z}_{+} .
\end{aligned}
$$

В [3] доказано (см. лемму 4), что при $\sigma_{-}=1, b_{i} \geqslant 0$ и $\sum_{j=0}^{\infty} b_{j}<+\infty$ система (15) обладает решением $h=\left(h_{0}, h_{1}, h_{2}, \ldots\right)$, причем

$$
h_{i} \geqslant 0, \quad i \in \mathbb{Z}_{+}, \quad h_{i} \leqslant \sum_{j=i}^{\infty} b_{j},
$$

и поэтому $h_{i} \rightarrow 0$ при $i \rightarrow \infty$.

Справедливо следующее утверждение.

Лемма 1. При условиях (17) и $\sigma_{+}<1$ решение системы (16) (которое является также решением системы (5)) будет обладать свойствами

$$
f_{i} \geqslant 0, \quad i \in \mathbb{Z}_{+}, \quad f_{i} \rightarrow 0, \quad i \rightarrow \infty .
$$

ДокАЗАтЕЛЬСтво. Очевидно, что для доказательства леммы достаточно показать, что соотношения (18) имеют место при $h_{i}=\sum_{j=i}^{\infty} b_{j}$.

Решение системы (16) в указанном случае можно представить в следующем виде (см. [4; п. 12]):

$$
f_{i}=\sum_{k=i}^{\infty} b_{k}+\sum_{j=0}^{i} \omega_{i-j} \sum_{k=j}^{\infty} b_{k}, \quad i \in \mathbb{Z}_{+},
$$

где последовательность $\omega=\left(\omega_{0}, \omega_{1}, \omega_{2}, \ldots\right)$ определяется из системы

$$
\omega_{i}=x_{i}+\sum_{j=0}^{i} x_{i-j} \omega_{j}, \quad i \in \mathbb{Z}_{+}
$$

и при $\sigma_{+}<1$ обладает свойствами

$$
\omega_{i} \geqslant 0, \quad i \in \mathbb{Z}_{+}, \quad \sum_{j=0}^{\infty} \omega_{j}<+\infty .
$$

Имеем $\sum_{j=i}^{\infty} b_{j} \rightarrow 0$ при $i \rightarrow \infty$. Для второй суммы правой части равенства (19) при $0 \leqslant p \leqslant i$ получаем

$$
\begin{aligned}
\sum_{j=0}^{i} \omega_{i-j} \sum_{k=j}^{\infty} b_{k} & =\sum_{j=0}^{p} \omega_{i-j} \sum_{k=j}^{\infty} b_{k}+\sum_{j=p}^{i} \omega_{i-j} \sum_{k=j}^{\infty} b_{k} \\
& \leqslant\|b\|_{l_{1}} \cdot \sum_{k=i-p}^{i} \omega_{k}+\sum_{j=p}^{i} \omega_{i-j} \sum_{k=j}^{\infty} b_{k}
\end{aligned}
$$


Для произвольного $\varepsilon>0$ выберем $p \in N$ и $i \in N$ так, чтобы

$$
\sum_{k=p}^{\infty} b_{k}<\frac{\varepsilon}{2\|\omega\|_{l_{1}}}, \quad \sum_{k=p}^{\infty} \omega_{k}<\frac{\varepsilon}{2\|b\|_{l_{1}}}, \quad i \geqslant 2 p .
$$

Тогда из (20) получаем

$$
0 \leqslant \sum_{j=0}^{i} \omega_{i-j} \sum_{k=j}^{\infty} b_{k}<\varepsilon
$$

что означает

$$
\sum_{j=0}^{i} \omega_{i-j} \sum_{k=j}^{\infty} b_{k} \rightarrow 0 \quad \text { при } \quad i \rightarrow \infty .
$$

Этим завершается доказательство леммы.

Теперь, учитывая оценки (11), можно утверждать, что при $0 \leqslant \lambda_{i} \leqslant 1, i \in \mathbb{Z}_{+}$, и $\sum_{k=0}^{\infty} b_{k}<+\infty$ решение $\rho=\left(\rho_{0}, \rho_{1}, \rho_{2}, \ldots\right)$ системы $(10)$, являющееся пределом соответствующих итераций, будет удовлетворять условиям

$$
\rho_{i} \geqslant 0, \quad i \in \mathbb{Z}_{+}, \quad \rho_{i} \rightarrow 0, \quad i \rightarrow \infty .
$$

Последние соотношения позволяют получить второе решение $\widetilde{\varphi}^{*}=\left(\widetilde{\varphi}_{0}^{*}, \widetilde{\varphi}_{1}^{*}, \widetilde{\varphi}_{2}^{*}, \ldots\right)$ системы (8), обладающее свойствами (9). Оно является пределом итераций

$$
\varphi_{i}^{(n+1)}=\left(1-\lambda_{i}^{*}\right) \eta_{i}^{*}+\lambda_{i}^{*} \sum_{j=0}^{\infty} a_{i-j} \varphi_{j}^{(n)}, \quad \varphi_{i}^{(0)}=0, \quad i \in \mathbb{Z}_{+}, \quad n=0,1,2 \ldots
$$

С использованием этих соотношений индукцией по $n$ можно доказать справедливость оценок $\varphi_{i}^{(n)} \leqslant \eta_{i}^{*}, i \in \mathbb{Z}_{+}, n=0,1,2, \ldots$, откуда в пределе при $n \rightarrow \infty$ получаем

$$
\widetilde{\varphi}_{i}^{*} \leqslant \eta_{i}^{*}, \quad i \in \mathbb{Z}_{+} .
$$

Таким образом, получили два решения системы (8): одним из решений $\varphi^{*}=\left(\varphi_{0}^{*}\right.$, $\left.\varphi_{1}^{*}, \varphi_{2}^{*}, \ldots\right)$ является последовательность $\varphi_{i}^{*}=\eta_{i}^{*}, i \in \mathbb{Z}_{+}$, так что $1 \leqslant \varphi_{1}^{*} \leqslant \varphi_{2}^{*} \leqslant \ldots ;$ второе решение $\widetilde{\varphi}^{*}=\left(\widetilde{\varphi}_{0}^{*}, \widetilde{\varphi}_{1}^{*}, \widetilde{\varphi}_{2}^{*}, \ldots\right)$ удовлетворяет условиям (9) и оценкам (21). Поэтому их разность, представляющую неотрицательную и ненулевую последовательность $\psi^{*}=\left(\psi_{0}^{*}, \psi_{1}^{*}, \psi_{2}^{*}, \ldots\right)$, где $\psi_{i}^{*}=\varphi_{i}^{*}-\widetilde{\varphi}_{i}^{*}, i \in \mathbb{Z}_{+}$, является решением системы

$$
\psi_{i}^{*}=\lambda_{i}^{*} \cdot \sum_{j=0}^{\infty} a_{i-j} \psi_{j}^{*}, \quad i \in \mathbb{Z}_{+} \cdot
$$

Итак, справедлива

ЛЕмма 2. При выполнении условий $(\alpha 1)-(\alpha 4)$ и ( $\beta 1)$ однородная система (22) обладает неотрицательным и ненулевым решением $\psi^{*}=\left(\psi_{0}^{*}, \psi_{1}^{*}, \psi_{2}^{*}, \ldots\right)$, причем

$$
\varphi_{i}^{*} \leqslant \eta_{i}^{*}, \quad i \in \mathbb{Z}_{+}, \quad \varphi_{i}^{*}-\eta_{i}^{*} \rightarrow 0, \quad i \rightarrow \infty,
$$

где $\eta^{*}$ - соответствующее решение системы (4) с той же матрицей $A=\left(a_{i-j}\right)$.

ЗАмЕчАНИЕ. Ввиду монотонности и ограниченности последовательности $\eta^{*}$ существует предел $\widetilde{\eta}=\lim _{k \rightarrow \infty} \eta_{k}^{*}$, причем $\widetilde{\eta}>1$. С другой стороны, $\lim _{k \rightarrow \infty} \widetilde{\varphi}_{k}^{*}=0$. Поэтому для произвольного $0<\tau<1$ существует номер $k_{0}$ такой, что $\psi_{k}^{*} \geqslant \tau$ при $k \geqslant k_{0}$. 
2. Неоднородная система и условия ее разрешимости. Теперь мы докажем, что при условиях $(\alpha 1)-(\alpha 4),(\beta 1)$ и $(\gamma 1),(\gamma 2)$ существует ограниченное решение системы (2), если дополнительно потребовать выполнение условия

$$
\sum_{j=-\infty}^{p} a_{j}>0, \quad p \in \mathbb{Z}
$$

Выберем последовательность $\lambda^{*}=\left(\lambda_{0}^{*}, \lambda_{1}^{*}, \lambda_{2}^{*}, \ldots\right)$ так, чтобы она удовлетворяла условиям $(\beta 2)$ и определялась через данную последовательность $b=\left(b_{0}, b_{1}, b_{2}, \ldots\right)$ системы (2). А именно, положим

$$
\lambda_{k}^{*} \stackrel{\text { def }}{=} 1-\mu b_{k}, \quad k \in \mathbb{Z}_{+},
$$

где $\mu$ - произвольное действительное число, $0<\mu<\left(\sum_{j=0}^{\infty} b_{j}\right)^{-1}$. Покажем, что если последовательность $b=\left(b_{0}, b_{1}, b_{2}, \ldots\right)$ удовлетворяет условиям $(\gamma 1),(\gamma 2)$, то определяемая посредством равенств (24) последовательность $\lambda^{*}$ будет обладать свойствами $(\beta 2)$ и, следовательно, соответствующая система $(22)$ будет иметь нетривиальное ограниченное решение $\psi^{*}$.

Действительно, из определения (24) непосредственно следует, что $0 \leqslant \lambda_{k}^{*} \leqslant 1$ и $1-\lambda_{k}^{*}=\mu \cdot b_{k}$, что равносильно условиям $(\beta 2)$.

Теперь рассмотрим ограниченные решения $\psi$ и $\psi^{*}$ систем $(3)$ и $(22)$, существующие в силу теоремы 1 и леммы 2 . Обозначая через $\Delta_{i}, i \in \mathbb{Z}_{+}$, разности $\Delta_{i}=\psi_{i}-\psi_{i}^{*}$ (очевидно, что $\Delta_{i} \geqslant 0, i \in \mathbb{Z}_{+}$), из (3) и (22) получим

$$
\Delta_{i}=\sigma_{i}+\lambda_{i} \cdot \sum_{j=0}^{\infty} a_{i-j} \Delta_{j}, \quad i \in \mathbb{Z}_{+}
$$

где величины $\sigma_{i}$ определяются посредством равенств

$$
\sigma_{i} \stackrel{\text { def }}{=}\left(\lambda_{i}-\lambda_{i}^{*}\right) \cdot \sum_{j=0}^{\infty} a_{i-j} \psi_{j}^{*}, \quad i \in \mathbb{Z}_{+} .
$$

Совокупность равенств (25) можно рассматривать как систему вида (2), имеющую неотрицательное ограниченное решение $\Delta=\left(\Delta_{0}, \Delta_{1}, \Delta_{2}, \ldots\right)$. Для величин $\sigma_{i}$ имеем оценки

$$
\sigma_{i} \geqslant\left(1-\lambda_{i}^{*}\right) \cdot \sum_{j=0}^{\infty} a_{i-j} \psi_{j}^{*}=\mu \cdot b_{i} \cdot \sum_{j=0}^{\infty} a_{i-j} \psi_{j}^{*}, \quad i \in \mathbb{Z}_{+},
$$

С другой стороны, учитывая замечание п. 1, можно оценить суммы в соотношениях (26):

$$
\sum_{j=0}^{\infty} a_{i-j} \psi_{j}^{*} \geqslant \sum_{j=k_{0}}^{\infty} a_{i-j} \psi_{j}^{*} \geqslant \tau \cdot \sum_{s=-\infty}^{i-k_{0}} a_{s} \geqslant \tau \cdot \sum_{s=-\infty}^{-k_{0}} a_{s} \equiv \xi,
$$

где $\xi>0$ ввиду условия $(\alpha 5)$.

Таким образом, получаем

$$
\sigma_{i} \geqslant \mu \xi \cdot b_{i}, \quad i \in \mathbb{Z}_{+},
$$


где $\mu \xi>0$. Соотношения (27) означают, что система (25) является мажорантой для системы

$$
\widetilde{\rho}_{i}=\mu \xi \cdot b_{i}+\lambda_{i} \cdot \sum_{j=0}^{\infty} a_{i-j} \widetilde{\rho}_{j}, \quad i \in \mathbb{Z}_{+} .
$$

Согласно вышеизложенным результатам и теореме 1 работы [2] последняя система обладает ограниченным решением $\widetilde{\rho}=\left(\widetilde{\rho}_{0}, \widetilde{\rho}_{1}, \widetilde{\rho}_{2}, \ldots\right)$. Следовательно, система $(2)$ также обладает ограниченным решением вида

$$
\varphi=\frac{1}{\mu \xi} \cdot \widetilde{\rho}, \quad \text { т.е. } \quad \varphi_{i}=\frac{1}{\mu \xi} \cdot \widetilde{\rho}_{i}, \quad i \in \mathbb{Z}_{+} .
$$

Итак, справедлива

Теорема 2. При выполнении условий $(\alpha 1)-(\alpha 5),(\beta 1),(\gamma 1),(\gamma 2)$ система (2) обладает неотрицательным ограниченным решением $\varphi=\left(\varphi_{0}, \varphi_{1}, \varphi_{2}, \ldots\right) \in m$.

ЗАмЕчАниЕ. Следует отметить, что при условиях $(\alpha 1)-(\alpha 5),(\beta 1)$ и $(\gamma 1),(\gamma 2)$ одновременно и однородная, и неоднородная системы вида (2) обладают ограниченными решениями.

В заключение автор выражает благодарность проф. Енгибаряну Н. Б. за обсуждение результатов работы.

\section{СПИСОК ЦИТИРОВАННОЙ ЛИТЕРАТУРЫ}

[1] Б. З. Вулих, Введение в функциональный анализ, ГИФМЛ, М., 1958.

[2] Л. В. Канторович, В. И. Крылов, Приближенные методы высшего анализа, ГИФМЛ, M., 1962.

[3] Л. Г. Арабаджян, "О дискретных уравнениях Винера-Хопфа", Матем. анализ и его прил., 1980, 43-48.

[4] Л. Г. Арабаджян, Н. Б. Енгибарян, "Уравнения в свертках и нелинейные функциональные уравнения", Итоги науки и техн. Сер. Мат. анал., 22, ВИНИТИ, М., 1984, $175-244$.

[5] N. B. Yengibarian, "Factorization of Markov chains", J. Theoret. Probab., 17:2 (2004), 459-481.

[6] L. G. Arabajian, M. T. Hakopian, "On one infinite algebraic system with a Toeplitz matrix", J. Integral Equations Math. Phys., 1:1 (1992), 32-43.

[7] Н. Б. Енгибарян, М.А. Мнацаканян, "Линейные алгебраические системы с теплицевыми матрицами", ЖК. вычисл. матем. и матем. физ., 17:5 (1977), 1102-1116.

[8] Л. Г. Арабаджян, "Об одном интегральном уравнении теории переноса в неоднородной среде", Дифферени. уравнения, 23:9 (1987), 1618-1622.

\section{Л. Г. Арабаджян}

Поступило

Институт математики НАН Республики Армении, 12.11.2008

Армянский государственный педагогический университет им. Х. Абовяна

E-mail: arabajyan@mail.ru 